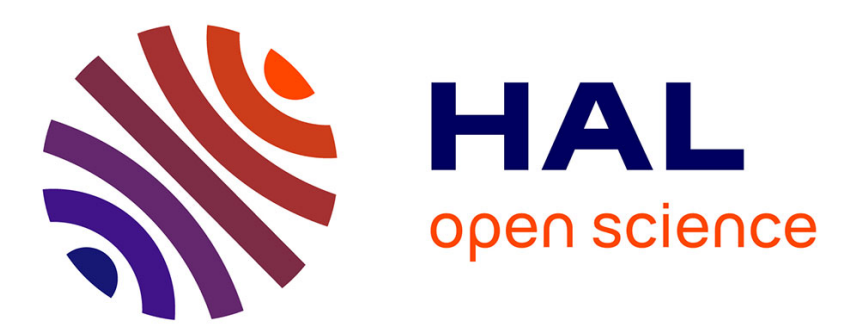

\title{
Negative Delta-T Noise in the Fractional Quantum Hall Effect
}

\author{
Jérôme Rech, T. Jonckheere, B. Grémaud, T. Martin
}

\section{To cite this version:}

Jérôme Rech, T. Jonckheere, B. Grémaud, T. Martin. Negative Delta-T Noise in the Fractional Quantum Hall Effect. Physical Review Letters, 2020, 125 (8), pp.086801. 10.1103/PhysRevLett.125.086801 . hal-02919436

\section{HAL Id: hal-02919436 https://hal.science/hal-02919436}

Submitted on 22 Aug 2020

HAL is a multi-disciplinary open access archive for the deposit and dissemination of scientific research documents, whether they are published or not. The documents may come from teaching and research institutions in France or abroad, or from public or private research centers.
L'archive ouverte pluridisciplinaire HAL, est destinée au dépôt et à la diffusion de documents scientifiques de niveau recherche, publiés ou non, émanant des établissements d'enseignement et de recherche français ou étrangers, des laboratoires publics ou privés. 


\title{
Negative Delta- $T$ Noise in the Fractional Quantum Hall Effect
}

\author{
J. Rech®, T. Jonckheere, B. Grémaud®, and T. Martin \\ Aix Marseille Univ, Université de Toulon, CNRS, CPT, Marseille, France
}

(Received 23 January 2020; accepted 28 July 2020; published 17 August 2020)

\begin{abstract}
We study the current correlations of fractional quantum Hall edges at the output of a quantum point contact subjected to a temperature gradient. This out-of-equilibrium situation gives rise to a form of temperature-activated shot noise, dubbed delta- $T$ noise. We show that the tunneling of Laughlin quasiparticles leads to a negative delta- $T$ noise, in stark contrast with electron tunneling. Moreover, varying the transmission of the quantum point contact or applying a voltage bias across the Hall bar may flip the sign of this noise contribution, yielding signatures that can be accessed experimentally.
\end{abstract}

DOI: 10.1103/PhysRevLett.125.086801

Introduction.-Noise is a fundamentally inescapable ingredient of any electronic device. While at first it may be regarded as a nuisance, it has now been broadly accepted as a key tool to improve our understanding of nanoscale conductors. Electronic noise is typically broken down into two contributions associated with different underlying physical phenomena. Thermal (or Johnson-Nyquist) noise is an equilibrium property, arising at a finite temperature from the thermal motion of electrons [1,2]. Shot noise manifests itself in a nonequilibrium situation, when current flows through a conductor, as a consequence of electrons being transmitted or reflected predominantly on a given side of the device [3].

Shot noise has taken a massive role in quantum mesoscopic physics, where it is commonly used to extract a great deal of information on the mechanisms of charge transfer (for a review see, e.g., [4,5]). While this nonequilibrium situation is typically achieved by imposing a bias voltage on the device, an intriguing alternative was recently uncovered. Indeed, one can in principle work at zero voltage bias and instead connect the sample to two reservoirs at different temperatures. This was realized experimentally using atomic-scale metallic junctions [6], where the authors showed that, while no net current was flowing through the device, as expected, a finite nonequilibrium noise signal was measured, which they dubbed "delta- $T$ noise." This previously undocumented source of noise, distinct from thermoelectric effects, actually corresponds to some form of temperatureactivated shot noise: it is purely thermal in origin, but only generated in a nonequilibrium situation. Its properties also present the same hybrid character, as this positive contribution to the total noise scales like the square of the temperature difference, while exhibiting the same dependence on the conductance as shot noise. Temperature-activated shot noise was exploited earlier to realize a local noise measurement in a current biased metallic conductor [7].
As it turns out, delta- $T$ noise is accurately described by the standard quantum theory of charge transport [6]. Remarkably, the scattering theory [8] does predict the appearance of this overlooked source of noise when a temperature difference alone is applied across a metallic junction. However, while the Fermi statistics of the charge carriers is accounted for within this formalism, interaction effects between electrons are discarded. We show here that delta- $T$ noise can be a unique tool to explore the properties of the charge carriers in a system where interactions and statistics play a significant role.

We propose to investigate the fate of delta- $T$ noise in a prototypical strongly correlated state, namely the edge states of the fractional quantum Hall effect (FQHE) [9-11]. In this system, excitations propagate along chiral edge modes, while the bulk is gapped. Transport through fractional quantum Hall edges has been extensively studied over the past few decades, both theoretically and experimentally. This interest is in part related to the bulkboundary correspondence, as edge transport reflects the topological nature of the bulk, revealing nontrivial properties unreachable through direct measurements. In particular, noise measurements in quantum point contact geometries (QPC) have been used experimentally to identify the fractional charge of the quasiparticle excitations in the bulk [12-14], and several proposals of more elaborate interferometric devices have been put forward as a way to probe their fractional statistics [15-23] with promising recent experimental results $[24,25]$. These considerations make the edge states of the FQHE a perfect testbed to examine delta- $T$ noise. Beyond the inherent interest in studying delta- $T$ noise in such systems, it may help better understanding charge and heat transport in situations where strong electronic correlations are operating [26]. The present Letter is an essential first step before more involved setups are considered, including, e.g., edge states involving neutral modes 
$[27,28]$ or more exotic fractions, where intriguing transport properties associated with thermal effects have been uncovered recently $[29,30]$.

Here we show that, for Laughlin fractions, the delta- $T$ noise signal is negative in the weak backscattering regime, a result which arises from the interplay of strong correlations and fractional statistics, and is directly accessible in modern experiments.

Delta-T noise.-We start by recalling results concerning delta- $T$ noise in noninteracting fermionic systems [6]. Consider a system of two fermionic reservoirs (with Fermi distribution $f_{1}$ and $f_{2}$, respectively) separated by a barrier of transmission $\mathcal{T}$. The fluctuations $\delta \hat{I}=\hat{I}-\langle\hat{I}\rangle$ of the current operator away from its average value are monitored using the zero-frequency current noise, defined as $S=2 \int d t\langle\delta \hat{I}(t) \delta \hat{I}(0)\rangle$. Within scattering theory, this quantity assumes a very general form [4]

$$
\begin{gathered}
S=\frac{e^{2}}{\pi} \int d E\left\{\mathcal{T}\left[f_{1}\left(1-f_{1}\right)+f_{2}\left(1-f_{2}\right)\right]\right. \\
\left.+\mathcal{T}(1-\mathcal{T})\left(f_{1}-f_{2}\right)^{2}\right\}
\end{gathered}
$$

where we considered for simplicity a single conduction channel and approximated the transmission coefficient by assuming it to be energy independent (setting $\hbar=1$ from this point onward). The first term corresponds to thermallike noise, while the last one is a nonequilibrium contribution. Let us now focus on the situation where no voltage bias is applied but the reservoirs are at different temperatures, yielding a temperature difference $\Delta T$, with an average temperature $\bar{T}$. Expanding for a small temperature difference leads to the following approximate expression for the current noise [6]

$$
S \approx 2 \frac{e^{2}}{\pi}\left[\mathcal{T} \bar{T}+\mathcal{T}(1-\mathcal{T}) \frac{\pi^{2}-6}{9}\left(\frac{\Delta T}{2 \bar{T}}\right)^{2} \bar{T}\right],
$$

where, along with the equilibrium thermal noise, another nonequilibrium component arises as a result of the temperature difference across the junction: delta- $T$ noise [31].

While these results apply to a very broad range of noninteracting devices, scattering theory is expected to fail in the fractional case calling for another formalism. It should, however, provide an accurate description of the properties of the system in the Fermi liquid regime, i.e., at filling factor $\nu=1$, or for a two-dimensional electron gas in the absence of a strong magnetic field (like the setup considered in [32] in the context of electron quantum optics). Our present derivation bridges the gap in providing a formalism that not only recovers the above Fermi-liquid result in the proper limit but also extends it to the case of quasiparticle tunneling.

Model.-The system considered here is a Hall bar (see Fig. 1), in the fractional quantum Hall regime, with a filling factor in the Laughlin sequence, i.e., $\nu=1 /(2 n+1)$

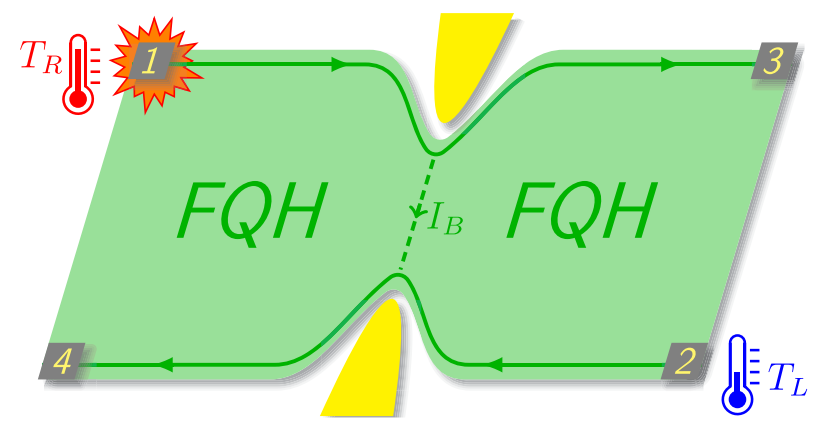

FIG. 1. Main setup: a quantum Hall bar equipped with a QPC connecting the chiral edge states of the FQH. The left-moving incoming edge is grounded at contact 2, with a temperature $T_{L}$ while the right-moving one is heated by contact 1 , reaching a temperature $T_{R}$. Relevant output currents are measured from contacts 3 and 4 .

$(n \in \mathbb{N})$. The edge states of such a sample are described in terms of a hydrodynamical model [33] by a chiral Luttinger liquid Hamiltonian of the form

$$
H_{0}=\frac{v_{F}}{4 \pi} \int d x\left[\left(\partial_{x} \phi_{R}\right)^{2}+\left(\partial_{x} \phi_{L}\right)^{2}\right],
$$

where $\phi_{R / L}$ are bosonic fields describing the right and left moving modes traveling along the edge with velocity $v_{F}$. They satisfy a Kac-Moody commutation relation of the form $\left[\phi_{R / L}(x), \phi_{R / L}(y)\right]= \pm i \pi \operatorname{Sgn}(x-y)$. These fields are directly related to the quasiparticle creation and annihilation operators through the bosonization identity, $\psi_{R / L}(x, t)=\left(U_{R / L} / \sqrt{2 \pi a}\right) e^{ \pm i k_{F} x} e^{-i \sqrt{\nu} \phi_{R / L}(x, t)}$, where $a$ is a short distance cutoff (typically the magnetic length), $k_{F}$ is the Fermi momentum, and $U_{R / L}$ are Klein factors. In particular, this identity gives us a direct connection between the bosonic fields and the quasiparticle density operator (and hence the current), as $\rho_{R / L}(x)=$ $\pm e(\sqrt{\nu} / 2 \pi) \partial_{x} \phi_{R / L}(x)$.

The Hall bar is further equipped with a quantum point contact, placed at position $x=0$, which allows tunneling between counterpropagating edges. In the weak backscattering regime, where quasiparticles are permitted to tunnel from one edge to the other through the bulk at the position of the QPC, this amounts to supplementing our Hamiltonian description with a tunneling term of the form

$$
H_{\mathrm{WB}}=\Gamma_{0} \psi_{R}^{\dagger}(0) \psi_{L}(0)+\text { H.c. }
$$

where we introduced the bare tunneling constant $\Gamma_{0}$.

Deriving current and noise.-We are primarily interested in the fluctuations of the current flowing between the edge states: the backscattered current $I_{B}(t)$. The latter is readily obtained from the tunneling Hamiltonian, Eq. (4), as the operator satisfying

$$
I_{B}(t)=-e \dot{N}_{R}=i e^{*} \Gamma_{0} \psi_{R}^{\dagger}(0, t) \psi_{L}(0, t)+\text { H.c. },
$$


with the effective charge $e^{*}=\nu e$. Since no voltage bias is applied across the Hall bar, it is easy to convince oneself [31] that no current flows between the counterpropagating edge states, i.e., $\left\langle I_{B}(t)\right\rangle=0$, independently of the respective temperature of the two edges.

Current-current correlations, however, do not vanish, as the finite temperature always lead to a nonzero contribution to the noise, through thermal fluctuations. More precisely, one can express the fluctuations of the backscattered current via the zero-frequency noise defined as

$$
\mathcal{S}_{B}=2 \int_{-\infty}^{+\infty} d \tau\left[\left\langle I_{B}(\tau) I_{B}(0)\right\rangle-\left\langle I_{B}(\tau)\right\rangle\left\langle I_{B}(0)\right\rangle\right]
$$

Using the Keldysh formalism, and relying on an expansion to the second order in the tunneling Hamiltonian, the zerofrequency noise can be written as [31]

$$
\mathcal{S}_{B}=\left(\frac{e^{*} \Gamma_{0}}{\pi a}\right)^{2} \int_{-\infty}^{+\infty} d \tau \exp \left[\nu \mathcal{G}_{R}(\tau)+\nu \mathcal{G}_{L}(\tau)\right]
$$

where we introduced the bosonic Green's functions $\mathcal{G}_{R / L}(\tau)$ typical of the chiral Luttinger liquid model of the FQHE edge states [5].

Let us now specifically consider the situation of a temperature gradient between the two input ports of the QPC. There, the above expression for the noise does not lead to a tractable analytic form as a function of the two relevant temperatures. Instead, we resort to a perturbative expansion in the temperature difference, in the spirit of the scattering theory results [6]. Following the parametrization in temperature $T_{R / L}=\bar{T} \pm(\Delta T / 2)$, we then expand the result of Eq. (7) in powers of $\Delta T$, noticing that, by symmetry under the exchange of reservoirs, the expansion contains no odd terms in the temperature difference. Up to fourth order in $\Delta T$, one has, after performing explicitly the resulting integrals

$$
\mathcal{S}_{B}=\mathcal{S}_{\mathrm{WB}}^{0}\left[1+\mathcal{C}_{\nu}^{(2)}\left(\frac{\Delta T}{2 \bar{T}}\right)^{2}+\mathcal{C}_{\nu}^{(4)}\left(\frac{\Delta T}{2 \bar{T}}\right)^{4}\right],
$$

where $\mathcal{S}_{\mathrm{WB}}^{0}$ is the usual thermal noise in the weak backscattering limit [up to order $O\left(\Gamma_{0}^{2}\right)$ ] at temperature $\bar{T}$, while $\mathcal{C}_{\nu}^{(2)}$ and $\mathcal{C}_{\nu}^{(4)}$ are numerical coefficients [31] depending only on the filling factor $\nu$. In particular, the leading order contribution has a prefactor

$$
\mathcal{C}_{\nu}^{(2)}=\nu\left\{\frac{\nu}{2 \nu+1}\left[\frac{\pi^{2}}{2}-\psi^{\prime}(\nu+1)\right]-1\right\}
$$

where $\psi^{\prime}(x)$ is the first derivative of the digamma function. Similarly, a closed analytic expression for the coefficient $\mathcal{C}_{\nu}^{(4)}$ is provided in the Supplemental Material [31].

Main results.-As it happens, in the special situation of a Hall system at filling factor $\nu=1$, our derivation yields

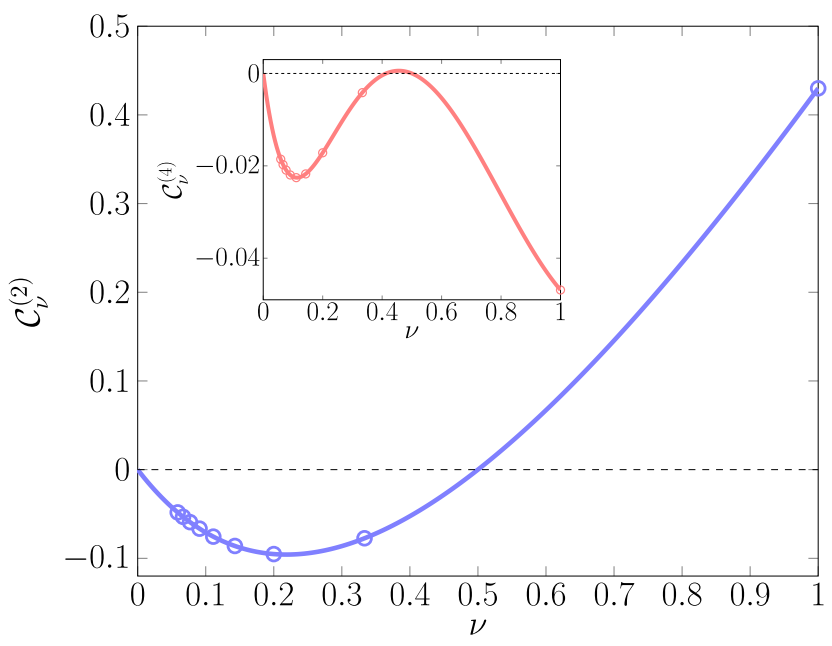

FIG. 2. Coefficients $\mathcal{C}_{\nu}^{(2)}$ (main) and $\mathcal{C}_{\nu}^{(4)}$ (inset) of the expansion of the noise in powers of the temperature difference [see Eq. (8)] as a function of the filling factor $\nu$. The circles correspond to the Laughlin fractions $\nu=1 /(2 n+1)$ for the first few values of $n=0,1, \ldots, 8$.

$\mathcal{C}_{\nu=1}^{(2)}=\left(\pi^{2}-6\right) / 9$, which matches with the noninteracting result obtained within scattering theory, and measured in metallic break junctions [6].

Our main results concern the fractional filling factors in the Laughlin sequence. We show in Fig. 2 the evolution of $\mathcal{C}_{\nu}^{(2)}$ (main figure) and $\mathcal{C}_{\nu}^{(4)}$ (inset) as a function of the filling factor $\nu$. From this, one readily sees that the second order term in the $\Delta T$ noise dominates over the fourth order one for all practical purposes, signaling that the expansion in $\Delta T$ is well controlled, despite the exotic power laws typical of the FQHE.

More importantly, our results presented in Fig. 2 suggest that the delta- $T$ noise is a negative contribution at leading order in $\Delta T$ for all filling factors in the Laughlin series. This represents a drastic difference, one of fundamental origin, with the noninteracting results where a positive correction was predicted and measured. Indeed, such negative contribution implies a reduction of the noise, which constitutes an intriguing result as noise sources typically tend to add up. A decrease in fluctuations signals a key role from interactions, suggesting that such negative delta- $T$ noise contribution is a direct signature of the correlation effects characteristic of the edge states of the FQHE.

Moreover, our results can be extended to the opposite regime of strong backscattering. This situation corresponds to a QPC near pinch-off, where the underlying Hall fluid is so depleted that only electrons can tunnel between the two halves of the Hall bar. We can repeat our derivation in this case [31] and show that it satisfies a duality transformation. The expression for the noise, Eq. (8), is thus only slightly altered as

$$
\mathcal{S}_{B}=\mathcal{S}_{\mathrm{SB}}^{0}\left[1+\mathcal{C}_{1 / \nu}^{(2)}\left(\frac{\Delta T}{2 \bar{T}}\right)^{2}+\mathcal{C}_{1 / \nu}^{(4)}\left(\frac{\Delta T}{2 \bar{T}}\right)^{4}\right],
$$


where $\mathcal{C}_{1 / \nu}^{(2)}$ and $\mathcal{C}_{1 / \nu}^{(4)}$ are readily obtained from the weak backscattering expressions upon substituting $\nu \rightarrow 1 / \nu$, for fractional filling factors within the Laughlin sequence $\nu=1 /(2 n+1)$. In particular, this means that in this regime of electron tunneling at the QPC, one recovers a positive signal for the delta- $T$ noise contribution, like in the noninteracting case albeit with very different coefficients.

This is important in two ways. First, this makes a potential detection all the more easy from an experimental standpoint, as the delta- $T$ noise component flips its sign when one adjusts the transmission of the QPC between the weak and the strong backscattering regimes. Second, this result may have significant implications for the physics at play here. Our results suggest that negative delta- $T$ noise can be directly tied to the tunneling of quasiparticles, as the sign of this noise contribution reverts back to the noninteracting case when electrons tunnel through the QPC. Similarly, one can show following somewhat similar calculations [31] that weakly coupled nonchiral Luttinger liquids (e.g., coupled nanowires or crossed nanotubes) cannot lead to negative backscattered noise when biased with a temperature difference. This underlines the importance of anyonic effects in the occurrence of negative delta$T$ noise, which cannot be viewed as purely due to interactions. Instead, this effect has more to do with the interplay of strong interaction and the fractional statistics of the anyons exchanged at the QPC. Actually, the appearance of negative current correlations in other setups involving fractional edge states has been associated with anyonic statistics [34,35]. In Ref. [35], it was argued that negative excess shot noise was associated with so-called topological vaccuum bubbles, an anyon process that has no counterpart for fermions. A similar mechanism involving the exchange of thermally excited anyons between the two edges at the QPC could be at play in the present device. Such a connection constitutes a fascinating incentive for future work in this direction.

Incidentally, in the (somewhat fictitious) situation of the filling factor $\nu=1 / 2$, one can show that all coefficients $\mathcal{C}_{\nu=1 / 2}^{(n)}$ reduce to zero, so that the delta- $T$ noise seems to exactly vanish in this case. This is actually an artifact of the weak backscattering limit. Using a refermionization approach [36,37], one can account for the tunneling at the QPC at all orders in $\Gamma_{0}$, and show that the lowest order contribution to the delta- $T$ noise is actually of order $\Gamma_{0}^{4}$, falling beyond our present perturbative treatment.

Voltage dependence.-For completeness, we now consider the case where both a bias voltage and a temperature difference are applied across the Hall bar. Here, one can still expand in powers of the temperature difference, generalizing the expression obtained in Eq. (8) to account for the finite voltage. In particular, the prefactor is now given by the voltage-dependent noise $\mathcal{S}_{\mathrm{WB}}^{0}(V)$, which coincides with the expected backscattering noise of a QPC at temperature $\bar{T}$ in the presence of a voltage bias

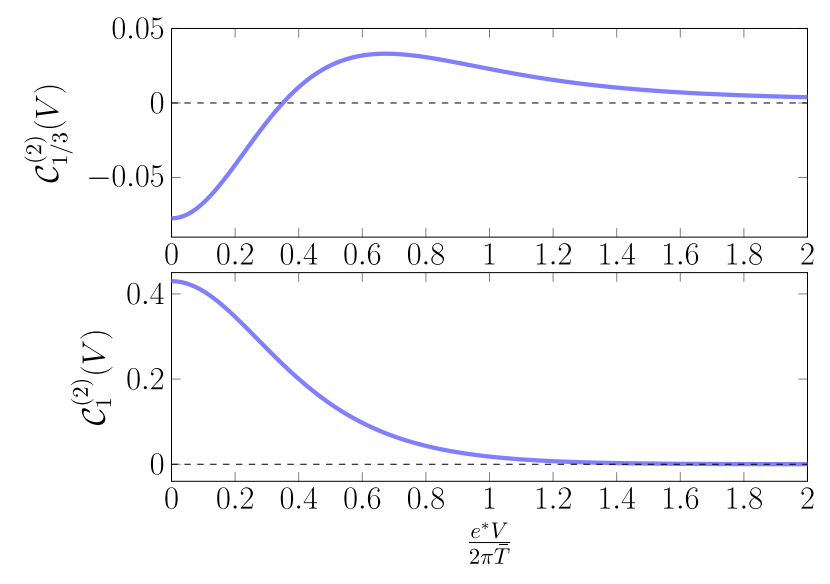

FIG. 3. Coefficients $\mathcal{C}_{1 / 3}^{(2)}(V)$ and $\mathcal{C}_{1}^{(2)}(V)$ of the expansion of the noise in powers of the temperature difference represented as a function of the applied bias across the Hall bar.

$V$ [36,38]. Similarly, the coefficients of the expansion in $\Delta T$ must now be replaced with voltage-dependent coefficients $\mathcal{C}_{\nu}^{(2)}(V)$ and $\mathcal{C}_{\nu}^{(4)}(V)$.

We show in Fig. 3 the evolution of the coefficients $\mathcal{C}_{1 / 3}^{(2)}(V)$ and $\mathcal{C}_{1}^{(2)}(V)$ of the leading contribution to the delta$T$ noise, obtained as a function of voltage for filling factor $\nu=1 / 3$ and $\nu=1$, respectively. Not only do these results recover the previously obtained values in the limit of vanishingly small voltage (compare with Fig. 2), they also predict that the coefficients of the expansion should tend toward zero for a large enough voltage bias, as expected. Even more importantly, this shows that while, in the noninteracting case, the sign of $\mathcal{C}_{1}^{(2)}(V)$ is fixed positive (as can be verified from the analytic expression [31]), in the fractional case, there exists a voltage scale where the coefficient $\mathcal{C}_{1 / 3}^{(2)}(V)$ changes sign.

Experimental realization.-We now look more carefully at a potential experimental realization that could reveal our results concerning the delta- $T$ noise. The considered setup would be a four-terminal device similar to the one schematically represented in Fig. 1. The working principle of such a device would be to heat up contact 1 , while leaving contact 2 at base temperature, and ensuring that no net current flows between edge states.

Experimentally, there is no way to directly access the fluctuations of the backscattered current. Instead, the measurement is performed on contacts 3 and 4, allowing us to probe the output currents $I_{3}$ and $I_{4}$ as well as the autocorrelations $\mathcal{S}_{33}$ and $\mathcal{S}_{44}$ and the cross-correlations $\mathcal{S}_{34}$. The latter should be favored since cross-correlations of the currents vanish at equilibrium, thus increasing the visibility of the relevant signal. Following standard calculations [39], one can show that the zero-frequency crossed correlations can be related to the backscattered noise [31], allowing us to introduce the reduced noise $\tilde{\mathcal{S}}=\mathcal{S}_{34}-4 \bar{T} G_{4}=-\mathcal{S}_{B}$, 
where $G_{4}=\left(\partial I_{4} / \partial V\right)$ is the differential conductance measured from contact 4 .

In practice, the average temperature $\bar{T}$ is not the most convenient quantity from an experimental standpoint. To circumvent this, it may be useful to slightly alter our parametrization, introducing instead $T_{L}=T_{\text {cold }}$ and $T_{R}=T_{\text {cold }}+\Delta T$, and measure the reduced noise in excess compared to the equilibrium situation. Rewriting our results with this prescription, we have, up to second order in $\Delta T$,

$$
\begin{aligned}
\Delta \tilde{\mathcal{S}}= & \tilde{\mathcal{S}}-\tilde{\mathcal{S}}^{\mathrm{eq}}=\tilde{\mathcal{S}}^{\mathrm{eq}}(2 \nu-1) \frac{\Delta T}{2 T_{\text {cold }}} \\
& \times\left[1+\left(\nu-1+\frac{\mathcal{C}_{\nu}^{(2)}}{2 \nu-1}\right) \frac{\Delta T}{2 T_{\text {cold }}}\right],
\end{aligned}
$$

where $\tilde{\mathcal{S}}^{\text {eq }}=-4 T_{\text {cold }} G_{4}^{0}$ (as $\mathcal{S}_{34}^{\text {eq }}=0$ ) and $G_{4}^{0}$ is the differential conductance in the absence of a temperature bias. This allows to extract the coefficient $\mathcal{C}_{\nu}^{(2)}$ directly from the experimental measurement of cross-correlations and conductance, with and without the temperature gradient.

In all generality, delta- $T$ noise may be partly eclipsed by thermoelectric effects. Indeed, as a result of a temperature difference, an electric current may develop even in the absence of any applied voltage, a phenomenon known as the Seebeck effect [40]. This thermally induced current can, in turn, lead to conventional shot noise. Following the results displayed in Fig. 3, for such thermoelectric effects to overshadow the negative contribution in $\Delta T$, one would need to generate a rather large voltage difference $V$, satisfying $e^{*} V \gtrsim 2.2 \bar{T}$. This is, however, unlikely to happen in quantum Hall devices. Not only is the bulk incompressible, and therefore expected not to contribute substantially to any relevant thermoelectric properties, but the edge states are also chiral and can only overlap at a single pointlike QPC, thus preserving particle-hole symmetry. Moreover, no equilibration is expected to take place over small enough distances (a few microns in relevant experiments) and the heat leakage into the bulk is similarly negligible for short enough edges, thus ruling out the impact of thermoelectric effects in realistic setups. Finally, it is also worth pointing out that in recent experiments on metallic break junctions [6], the thermoelectric contribution was shown to be orders of magnitude smaller than the delta$T$ noise.

Conclusions. - We have studied the peculiar signatures of a temperature difference across a QPC, showing that it leads to a negative contribution to the noise associated with the tunneling of Laughlin quasiparticles. We have characterized this so-called delta- $T$ noise in the weak and strong backscattering regimes and investigated its dependence on voltage bias. We argued that it is readily accessible in current experiments involving fractional edge states.

This work offers many interesting perspectives, accounting more carefully for equilibration (important for long edges) or thermoelectric effects (e.g., associated with an extended QPC [41]). The most intriguing one concerns the extension to more exotic states, beyond the Laughlin filling factors, where many new fascinating effects should occur as a consequence of the more complex structure of the edge states (and in particular, the presence of neutral modes $[28,42])$.

We are grateful to L. Raymond, D. C. Glattli, G. Fève, H. Bartolomei, and P. Degiovanni for useful discussions. The project leading to this publication has received funding from Excellence Initiative of Aix-Marseille UniversityA*MIDEX, a French "Investissements d'Avenir" program (Grant No. AMX-19-IET-009).

[1] J. Johnson, Thermal agitation of electricity in conductors, Nature (London) 119, 50 (1927).

[2] H. Nyquist, Thermal agitation of electric charge in conductors, Phys. Rev. 32, 110 (1928).

[3] W. Schottky, Über spontane stromschwankungen in verschiedenen elektrizitätsleitern, Ann. Phys. (Berlin) 362, 541 (1918).

[4] Y. Blanter and M. Büttiker, Shot noise in mesoscopic conductors, Phys. Rep. 336, 1 (2000).

[5] T. Martin, Noise in mesoscopic physics, in Nanophysics: Coherence and Transport, Les Houches, Session LXXXI, edited by H. Bouchiat, Y. Gefen, S. Guéron, G. Montambaux, and J. Dalibard (Elsevier, Amsterdam, 2005), p. 283.

[6] O. Lumbroso, L. Simine, A. Nitzan, D. Segal, and O. Tal, Electronic noise due to temperature differences in atomicscale junction, Nature (London) 562, 240 (2018).

[7] E. S. Tikhonov, D. V. Shovkun, D. Ercolani, F. Rossella, M. Rocci, L. Sorba, S. Roddaro, and V.S. Khrapai, Local noise in a diffusive conductor, Sci. Rep. 6, 30621 (2016).

[8] R. Landauer, Spatial variation of currents and fields due to localized scatterers in metallic conduction, IBM J. Res. Dev. 1, 223 (1957).

[9] R. B. Laughlin, Nobel lecture: Fractional quantization, Rev. Mod. Phys. 71, 863 (1999).

[10] H. L. Stormer, Nobel lecture: The fractional quantum Hall effect, Rev. Mod. Phys. 71, 875 (1999).

[11] D. C. Tsui, Nobel lecture: Interplay of disorder and interaction in two-dimensional electron gas in intense magnetic fields, Rev. Mod. Phys. 71, 891 (1999).

[12] L. Saminadayar, D. C. Glattli, Y. Jin, and B. Etienne, Observation of the $e / 3$ Fractionally Charged Laughlin Quasiparticle, Phys. Rev. Lett. 79, 2526 (1997).

[13] R. de Picciotto, M. Reznikov, M. Heiblum, V. Umansky, G. Bunin, and D. Mahalu, Direct observation of a fractional charge, Nature (London) 389, 162 (1997).

[14] M. Dolev, M. Heiblum, V. Umansky, A. Stern, and D. Mahalu, Observation of a quarter of an electron charge at the $\nu=5 / 2$ quantum Hall state, Nature (London) 452, 829 (2008).

[15] C. de C. Chamon, D. E. Freed, S. A. Kivelson, S. L. Sondhi, and X.G. Wen, Two point-contact interferometer for quantum Hall systems, Phys. Rev. B 55, 2331 (1997). 
[16] I. Safi, P. Devillard, and T. Martin, Partition Noise and Statistics in the Fractional Quantum Hall Effect, Phys. Rev. Lett. 86, 4628 (2001).

[17] S. Vishveshwara, Revisiting the Hanbury Brown-Twiss Setup for Fractional Statistics, Phys. Rev. Lett. 91, 196803 (2003).

[18] C. L. Kane, Telegraph Noise and Fractional Statistics in the Quantum Hall Effect, Phys. Rev. Lett. 90, 226802 (2003).

[19] E.-A. Kim, M. Lawler, S. Vishveshwara, and E. Fradkin, Signatures of Fractional Statistics in Noise Experiments in Quantum Hall Fluids, Phys. Rev. Lett. 95, 176402 (2005).

[20] F. E. Camino, W. Zhou, and V. J. Goldman, Realization of a Laughlin quasiparticle interferometer: Observation of fractional statistics, Phys. Rev. B 72, 075342 (2005).

[21] K. T. Law, D. E. Feldman, and Y. Gefen, Electronic Mach-Zehnder interferometer as a tool to probe fractional statistics, Phys. Rev. B 74, 045319 (2006).

[22] G. Campagnano, O. Zilberberg, I. V. Gornyi, D. E. Feldman, A. C. Potter, and Y. Gefen, Hanbury Brown-Twiss Interference of Anyons, Phys. Rev. Lett. 109, 106802 (2012).

[23] G. Campagnano, O. Zilberberg, I. V. Gornyi, and Y. Gefen, Hanbury brown and twiss correlations in quantum Hall systems, Phys. Rev. B 88, 235415 (2013).

[24] H. Bartolomei, M. Kumar, R. Bisognin, A. Marguerite, J.-M. Berroir, E. Bocquillon, B. Plaçais, A. Cavanna, Q. Dong, U. Gennser et al., Fractional statistics in anyon collisions, Science 368, 173 (2020).

[25] J. Nakamura, S. Liang, G. C. Gardner, and M. J. Manfra, Direct observation of anyonic braiding statistics at the $\nu=1 / 3$ fractional quantum Hall state, arXiv:2006.14115.

[26] E. Sivre, H. Duprez, A. Anthore, A. Aassime, F. D. Parmentier, A. Cavanna, A. Ouerghi, U. Gennser, and F. Pierre, Electronic heat flow and thermal shot noise in quantum circuits, Nat. Commun. 10, 5638 (2019).

[27] A. Bid, N. Ofek, H. Inoue, M. Heiblum, C. Kane, V. Umansky, and D. Mahalu, Observation of neutral modes in the fractional quantum Hall regime, Nature (London) 466, 585 (2010).

[28] O. Shtanko, K. Snizhko, and V. Cheianov, Nonequilibrium noise in transport across a tunneling contact between $\nu=\frac{2}{3}$ fractional quantum Hall edges, Phys. Rev. B 89, 125104 (2014).
[29] M. Banerjee, M. Heiblum, A. Rosenblatt, Y. Oreg, D. Feldman, A. Stern, and V. Umansky, Observed quantization of anyonic heat flow, Nature (London) 545, 75 (2017).

[30] M. Banerjee, M. Heiblum, V. Umansky, D. Feldman, Y. Oreg, and A. Stern, Observation of half-integer thermal Hall conductance, Nature (London) 559, 205 (2018).

[31] See the Supplemental Material at http://link.aps.org/ supplemental/10.1103/PhysRevLett.125.086801 for further details of the derivation.

[32] J. Dubois, T. Jullien, F. Portier, P. Roche, A. Cavanna, Y. Jin, W. Wegscheider, P. Roulleau, and D. C. Glattli, Minimalexcitation states for electron quantum optics using levitons, Nature (London) 502, 659 (2013).

[33] X. G. Wen, Topological orders and edge excitations in fqh states, Adv. Phys. 44, 405 (1995).

[34] B. Rosenow, I. P. Levkivskyi, and B. I. Halperin, Current Correlations from a Mesoscopic Anyon Collider, Phys. Rev. Lett. 116, 156802 (2016).

[35] B. Lee, C. Han, and H.-S. Sim, Negative Excess Shot Noise by Anyon Braiding, Phys. Rev. Lett. 123, 016803 (2019).

[36] C. de C. Chamon, D. E. Freed, and X. G. Wen, Nonequilibrium noise in chiral Luttinger liquids, Phys. Rev. B 53, 4033 (1996).

[37] P. Sharma and C. Chamon, Adiabatic charge and spin transport in interacting quantum wires, Phys. Rev. B 68, 035321 (2003).

[38] C. L. Kane and M. P. A. Fisher, Nonequilibrium Noise and Fractional Charge in the Quantum Hall Effect, Phys. Rev. Lett. 72, 724 (1994).

[39] G. Campagnano, P. Lucignano, and D. Giuliano, Chirality and current-current correlation in fractional quantum Hall systems, Phys. Rev. B 93, 075441 (2016).

[40] T. M. Tritt and M. A. Subramanian, Thermoelectric materials, phenomena, and applications: A bird's eye view, MRS Bull. 31, 188 (2006).

[41] L. Vannucci, F. Ronetti, G. Dolcetto, M. Carrega, and M. Sassetti, Interference-induced thermoelectric switching and heat rectification in quantum Hall junctions, Phys. Rev. B 92, 075446 (2015).

[42] D. Ferraro, A. Braggio, N. Magnoli, and M. Sassetti, Neutral modes edge state dynamics through quantum point contacts, New J. Phys. 12, 013012 (2010). 